\title{
SPECTROSCOPY OF LUMINOUS INFRARED GALAXIES
}

\author{
S. VEILLEUX \\ University of Maryland \\ College Park, MD 20742 USA
}

\begin{abstract}
A review of recent optical and infrared spectroscopic resu si on luminous infrared galaxies is presented. The main emphasis is on the ultraluminous objects. Possible correlations with infrared luminosity are identified. These results are used to constrain the nature of the dominant energy source in luminous infrared galaxies, and to test whether these objects may represent an evolutionary phase between starburst galaxies and active galactic nuclei.
\end{abstract}

\section{Introduction}

Ultraluminous infrared galaxies (hereafter ULIGs: $L_{\mathrm{ir}}{ }^{1} \geq 10^{12} L_{\odot} ; H_{0}=75$ $\mathrm{km} \mathrm{s}^{-1} \mathrm{Mpc}^{-1}, q_{0}=0.5$ ) are an important class of extragalactic objects. Not only are they among the most luminous objects in the universe, with bolometric luminosities higher than many quasars, but they also dominate the top end of the galaxy luminosity function, with space densities that exceed those of optically selected quasars in the same luminosity range (Soifer et al. 1987). While exceptional in comparison with the majority of galaxies, ULIGs may have broader significance as representatives of a brief phase in the formation of massive galaxies and/or the genesis of active galactic nuclei (AGN; see review by Sanders \& Mirabel 1996).

Assessing the relative importance of an AGN and massive stars for powering ULIGs is essential for understanding these objects as massive galaxies and/or quasars in formation, and for testing suggestions of an evolutionary connection between starburst and AGN phenomena. Considerable effort has therefore been invested in recent years in trying to determine the domi-

${ }^{1} L_{\text {ir }} \equiv L(8-1000 \mu \mathrm{m})$, computed from the observed infrared fluxes in all four IRAS bands according to the prescription outlined in Perault (1987); see also Sanders \& Mirabel (1996)

J.E. Barnes and D.B. Sanders (eds.), Galaxy Interactions at Low and High Redshift, 295-301.

(C) 1999 IAU. Printed in the Netherlands. 
nant energy source in these objects. In $\S 2$ and $\S 3$, we summarize the results derived from ground-based optical and near-infrared spectroscopy. In $\S 4$, these results are compared with those obtained at longer wavelengths with ISO.

\section{Optical Spectroscopy}

All ULIGs present a large concentration of activity in their nuclei, including strong emission lines characteristic of a starbursting stellar population and in some cases, broad or high-ionization emission lines that suggest the presence of a powerful AGN (e.g., Sanders et al. 1988; Leech et al. 1989; Armus et al. 1989; Allen et al. 1991; Ashby et al. 1992, 1995; Vader et al. 1993; Veilleux et al. 1995; Clements et al. 1996). Here, we focus our discussion on the results from our own optical study of an unbiased subset of 45 ULIGs from the ' 1 Jy' sample (Kim 1995; Kim \& Sanders 1998; Kim, Veilleux, \& Sanders 1998).

Using several emission-line diagnostic diagrams (e.g., Veilleux \& Osterbrock 1987; Osterbrock et al. 1992; Dopita \& Sutherland 1995), these objects were classified as H II galaxies (star-forming galaxies with spectra resembling those of normal H II regions), LINERs (Low-Ionization Nuclear Emission-Line Regions; Heckman 1980), Seyfert 2s (AGN with strong lowand high-ionization lines), and Seyfert 1s (AGN with broad, quasar-like recombination lines; FWHM $\gtrsim 2,000 \mathrm{~km} \mathrm{~s}^{-1}$ ). These data were then combined with the results from a previous study of primarily lower luminosity infrared galaxies (Veilleux et al. 1995) to examine the spectral properties of luminous infrared galaxies over the range $L_{\mathrm{ir}} \approx 10^{10.5}-10^{12.8} L_{\odot}$. We find that the fraction of luminous infrared galaxies with Seyfert characteristics increases rapidly with increasing $L_{\mathrm{ir}}$. A rough inspection of the rest of the ' $1 \mathrm{Jy}$ ' sample seems to confirm this trend (Fig. 1). For $L_{\mathrm{ir}}>10^{12.3} L_{\odot}$, we find that nearly $60 \%$ of the ULIGs are optically classified as Seyfert $1 \mathrm{~s}$ or $2 \mathrm{~s}$.

However, even in cases where an AGN is present, starburst activity may contribute a large fraction of the total bolometric luminosity. This is evident from our spectra of the circumnuclear regions in these objects which indicate the presence of a young $\left(\sim 10^{7}\right.$ year $)$ stellar population at all radii. We discuss in the next section how to further constrain the nature of the dominant energy source in these objects. 


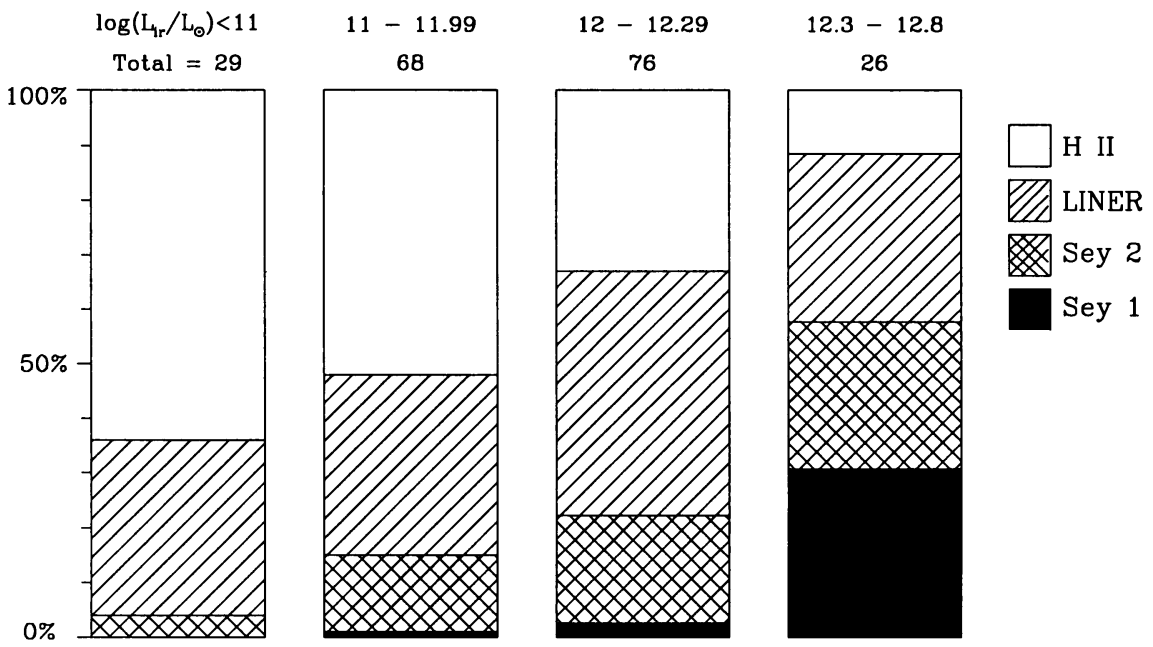

Figure 1. Optical spectral classification as a function of infrared luminosity for the objects in the ' $1 \mathrm{Jy}$ ' sample and the luminous infrared galaxies in the Bright Galaxy Survey (Veilleux et al. 1995, 1997b).

\section{Near-Infrared Spectroscopy}

Recent progress in infrared detector technology now provides a new approach to address the issue of the energy source in ULIGs. Infrared spectroscopy has the potential to more deeply probe the cores of ULIGs: for example, the extinction coefficient in the $\mathrm{K}$ band is nearly 10 times smaller than at optical wavelengths. This technique has proven very useful in the study of highly reddened broad-line regions (BLRs) in intermediate Seyferts (1.8's and 1.9's; Goodrich 1990; Rix et al. 1990) and has also had success finding obscured BLRs in some optically classified Seyfert 2 and radio galaxies (Blanco et al. 1990; Goodrich et al. 1994; Hill et al. 1996; Ruiz et al. 1994; Veilleux et al. 1997a). Low-resolution infrared spectra of a handful of ULIGs have shown tantalizing evidence for hidden BLRs (Armus et al. 1995; DePoy et al. 1987; Hines 1991; Nakajima et al. 1991a, b). Spectropolarimetry has lent support to some of these findings (Hines 1991; Hines et al. 1995; Hines \& Wills 1993; Hough et al. 1991; Young et al. 1993). However, more recent spectroscopy of a large sample of galaxies with relatively low infrared 
luminosity did not reveal any new, previously unknown BLRs (Goldader et al. 1995, 1997a, b).

In an attempt to verify these results among ULIGs of higher luminosity, our group recently carried out a near-infrared spectroscopic study of the nuclear regions of 25 objects from the ' $1 \mathrm{Jy}$ ' sample (Veilleux et al. 1997b). These objects were selected for their lack of BLRs at optical wavelengths. The redshift range of our subsample $(z \sim 0.1-0.2)$ allowed us to search for broad-line emission from the strong $\mathrm{Pa} \alpha \lambda 1.8751 \mu \mathrm{m}$ feature (intrinsically 12 times stronger than the $\operatorname{Br} \gamma$ line used by Goldader et al. 1995) and for the high-ionization [Si VI] $\lambda 1.962 \mu \mathrm{m}$ emission line, two powerful AGN diagnostic lines which are generally inaccessible in lower redshift objects. The results of this study can be summarized as follows:

1. $70-90 \%$ (7 or possibly 9 out of 10 ) of the optically classified Seyfert $2 \mathrm{~s}$ in our sample show signs of AGN activity at rest wavelengths $~$ $2 \mu \mathrm{m}$ (i.e. BLR or [Si VI] emission; Fig. 2). The optical and nearinfrared results taken together, therefore suggest that the total fraction of objects in the 1 Jy sample with signs of bonafide AGN is $\sim 25$ $30 \%$, but reaches $\sim 50 \%$ for those objects with $\log \left[L_{\text {ir }} / L_{\odot}\right]>12.3$.

2. All 6 'warm' $(F[25 \mu \mathrm{m}] / F[60 \mu \mathrm{m}]>0.2)$ optically classified Seyfert 2 galaxies in our sample show either obscured BLRs or [Si VI] emission at near-infrared wavelengths, and present large $\mathrm{Pa} \alpha$-to-infrared luminosity ratios. These results suggest that the screen of dust obscuring the cores of 'warm' Seyfert 2 ULIGs is often optically thin at $2 \mu \mathrm{m}$.

3. No obvious signs of an obscured BLR or strong [Si VI] emission are detected in any of the 15 optically classified LINERs and H II galaxies in our sample. The Pa $\alpha$-to-IR luminosity ratios and IRAS colors of the LINERs suggest that dust obscuration is significant in these objects and may be sufficient to hide a central AGN. The H II galaxies in our sample span a wide range of IRAS colors and are strong emitters of narrow $\mathrm{Pa} \alpha$. Dust obscuration therefore appears to be relatively unimportant in many of these objects.

4. No correlation is found between narrow-line extinctions and the presence of an obscured BLR. The measurements derived from the narrow lines probably only reflect the properties of the circumnuclear gas rather than that of the dusty screen directly in front of the nucleus.

5. The obscured BLRs in our galaxies have dereddened broad-line luminosities which are similar to those of optically selected quasars of comparable bolometric luminosity. This is not what would be expected if these ULIGs were powered predominantly by a starburst. Most of the bolometric luminosity in these objects therefore appears to be powered by the same mechanism as that in optical quasars, namely mass accre- 

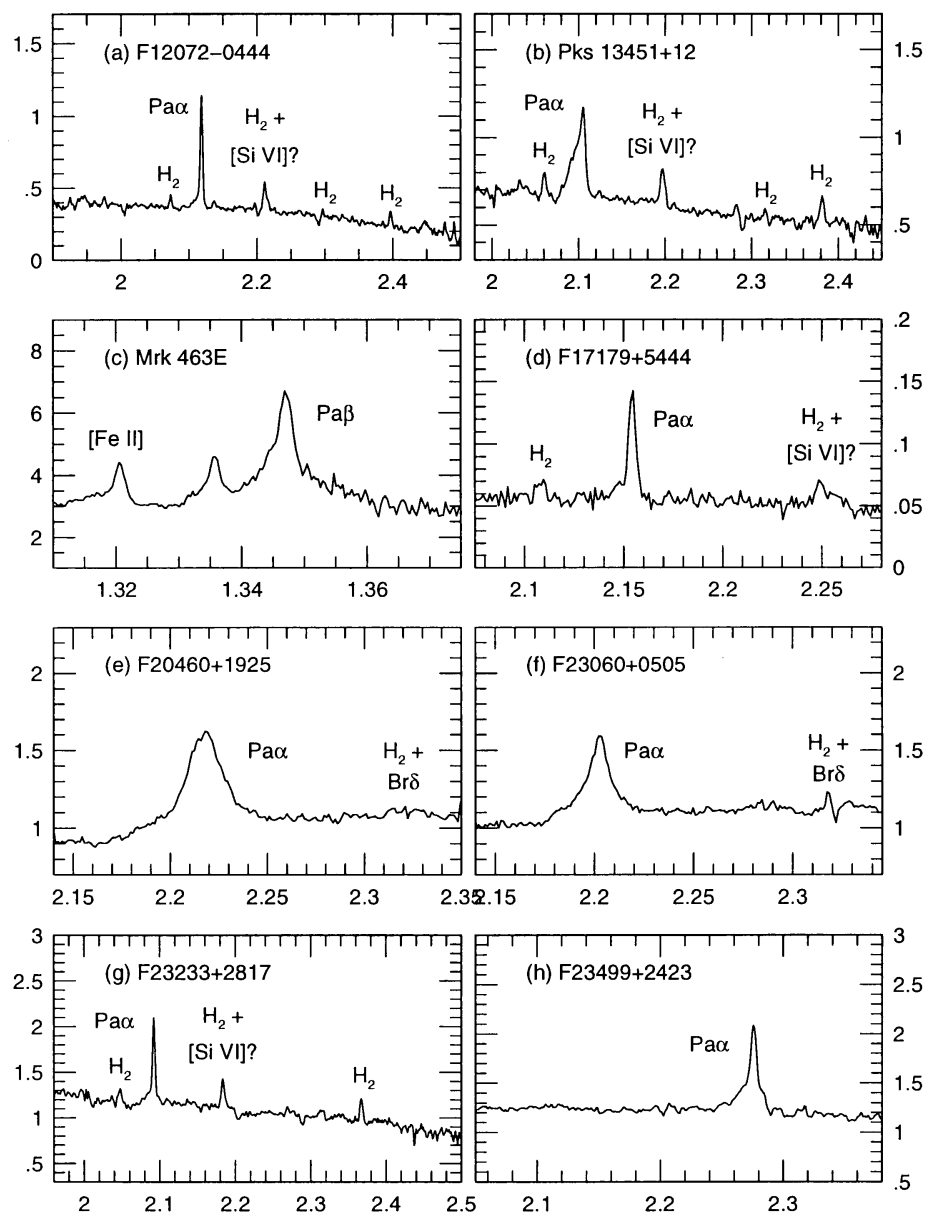

Figure 2. Reduced near-infrared spectra of 8 ultraluminous infrared galaxies optically

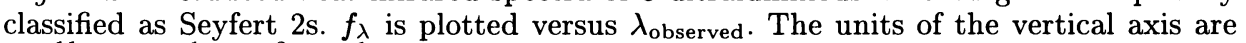
$10^{-11} \mathrm{ergs} \mathrm{s}^{-1} \mathrm{~cm}^{-2} \mu \mathrm{m}^{-1}$, while the wavelength scale is in $\mu \mathrm{m}$. Broad $\mathrm{Pa} \alpha$ or $\mathrm{Pa} \beta$ emission with FWHM $\gtrsim 2,000 \mathrm{~km} \mathrm{~s}^{-1}$ is detected in Pks $1345+12$, Mrk 463E, F20460+1925, F23060+0505, and F23499+2423. Possible [Si VI] detections are also indicated in these figures. (Veilleux et al. 1997b) 
tion onto a supermassive black hole. The fraction of quasar-dominated ULIGs appears to increase with increasing infrared luminosity.

\section{Comparisons with Recent ISO Results}

The results from the ISO Central Program on ultraluminous infrared galaxies have recently been published (Genzel et al. 1998 and references therein). At first, the $I S O$ results appear to contradict the results obtained from the ground: $70-80 \%$ of the ULIGs examined by ISO are predominantly powered by a starburst, while $20-30 \%$ are powered by a central AGN. However, a closer inspection of the ISO sample shows that nearly all of the objects in this sample have infrared luminosities less than $10^{12.3} L_{\odot}$ and therefore fall within the second highest luminosity bin in Figure 1. At these lower luminosities, our ground-based survey indicates that the percentage of ULIGs with signs of AGN is only $20-30 \%$, i.e. comparable to the fraction of ULIGs in the ISO sample which are powered by a central AGN. Unfortunately, objects with $\log \left[L_{\text {ir }} / L_{\odot}\right]>12.3$ are relatively faint and difficult to observe with $I S O$. Only four such objects were included in the ISO Central Program. Of these, two appear to be powered predominantly by an AGN (Mrk 231 and F23060+0505; Genzel et al. 1998). The number of objects is clearly insufficient to draw reliable conclusions. However, these preliminary results are consistent with the high percentage $(\sim$ $50 \%$ ) of high-luminosity objects that show signs of an AGN at optical and near-infrared wavelengths.

If confirmed by more exhaustive studies, this trend with infrared luminosity might be a crucial element to our understanding of the origin and evolution of ULIGs. In one possible scenario, ULIGs represent a transition phase in the evolution of powerful nuclear starbursts into optical quasars (Sanders et al. 1988). The starburst is triggered by the tidal interaction between two gas-rich galaxies and, as the merger takes place, a large amount of gas is funneled toward the center, and the luminosity of the system increases dramatically (e.g., Mihos \& Hernquist 1994). The physical conditions become favorable to the formation of a central massive black hole or to the fueling of a preexisting black hole; an active galactic nucleus results. According to this scenario, low-luminosity starburst-dominated ULIGs are the parent population of high-luminosity quasar-dominated ULIGs and optical quasars. Clearly, it will be important to quantify the possible spectroscopic trends with infrared luminosity among ULIGs to test this evolutionary scenario and shed new light on the starburst and AGN phenomena. 


\section{Acknowledgements}

The ground-based study discussed in this paper was done in collaboration with Drs. D. B. Sanders and D.-C. Kim. The author gratefully acknowledges the financial support of NASA through grant number HF-1039.0192A awarded by the Space Telescope Science Institute which is operated by the AURA, Inc. for NASA under contract No. NAS5-26555.

\section{References}

Allen, D. A., Norris, R. P., Meadows, V. S., \& Roche, P. F. 1991, MNRAS, 248, 528

Armus L., Heckman, T. M., \& Miley, G. K. 1989, Ap. J., 347, 727

Armus, L., Neugebauer, G., Soifer, B. T., \& Matthews, K. 1995, A. J., 110, 2610

Ashby, M., Houck, J. R., \& Hacking, P. B. 1992, Ap. J., 10, 980

Ashby, M., Houck, J. R., \& Matthews, K. 1995, Ap. J., 447, 545

Blanco, P. R., Ward, M. J., \& Wright, G. S. 1990, $M N R A S, \mathbf{2 4 2}, 4 \mathrm{P}$

Clements, D. L., et al. 1996, M. N.R. A. S., 279, 459

DePoy, D. L., Becklin, E. E., \& Geballe, T. R. 1987, Ap. J., 316, L63

Dopita, M. A., \& Sutherland, R. S. 1995, Ap. J., 455, 468

Genzel, R. et al. 1998, Ap. J., 498, 579

Goldader, J. D., Joseph, R. D., Doyon, R., \& Sanders, D. B. 1995, Ap. J., 444, 97

—. 1997a, Ap. J. Suppl., 108, 449 1997b, Ap. J., 472, 104

Goodrich, R. W. 1990, Ap. J., 355, 88

Goodrich, R. W., Veilleux, S., \& Hill, G. J. 1994, Ap. J., 422, 521

Heckman, T. M. 1980, A. \& A., 87, 142

Hill, G. J., Goodrich, R. W., \& DePoy, D. L. 1996, Ap. J., 462, 163

Hines, D. C. $1991, A p$. J., 374, L9

Hines, D. C., et al. 1995, Ap. J., 450, L1

Hines, D. C., \& Wills, B. J. 1993, Ap. J., 415, 82

Hough, J. H., et al. 1991, Ap. J., 372, 478

Kim, D.-C. 1995, Ph.D. Thesis, University of Hawaii

Kim, D.-C., \& Sanders, D. B. 1998, Ap. J. Suppl., 119, in press

Kim, D.-C., Veilleux, S., \& Sanders, D. B. 1998, Ap. J., in press

Leech, K. J., et al. 1989, M. N. R. A. S., 240, 349

Mihos, J. C., \& Hernquist, L. 1994, Ap. J., 431, L9

Nakajima, T., Carleton, N. P., \& Nishida, M. 1991a, Ap. J., 375, L1

Nakajima, T., Kawara, K., Nishida, M., \& Gregory, B. 1991b, Ap. J., 373, 452

Osterbrock, D. E., Tran, H. D., \& Veilleux, S. 1992, Ap. J., 389, 196

Perault, M. 1987, Ph.D. Thesis, University of Paris

Rix, H.-W., Carleton, N. P., Rieke, G., \& Rieke, M. 1990, Ap. J., 363, 480

Ruiz, M., Rieke, G. H., \& Schmidt, G. D. 1994, Ap. J., 423, 608

Sanders, D. B., \& Mirabel, I. F. 1996, AR\&A, 34, 725

Sanders, D. B., et al. 1988, Ap. J., 325, 74

Soifer, B. T., et al. 1987, Ap. J., 320, 238

Vader, J. P., Frogel, J. A., Terndrup, D. M., \& Heisler, C. A. 1993, A. J., 106, 1743

Veilleux, S., Goodrich, R. W., \& Hill, G. J. 1997a, Ap. J., 477, 631

Veilleux, S., et al. 1995, Ap. J. Suppl., 98, 171

Veilleux, S., \& Osterbrock, D. E. 1987, Ap. J. Suppl., 63, 295

Veilleux, S., Sanders, D. B., \& Kim, D.-C. 1997b, Ap. J., 484, 92

Young, S., et al. 1993, M. N. R. A. S., 260, L1 